\title{
ENFERMEDADES INFECCIOSAS DESATENDIDAS: UN PERMANENTE RETO PARA LA SALUD PÚBLICA Y LA EQUIDAD EN EL PERÚ
}

\author{
NEGLECTED INFECTIOUS DISEASES: AN ONGOING CHALLENGE FOR \\ PUBLIC HEALTH AND EQUITY IN PERU
}

César Cabezas-Sánchez ${ }^{1, a}$

\author{
Centro Nacional de Salud Pública. Instituto Nacional de Salud. Lima, Perú. \\ Médico infectólogo-tropicalista. \\ Recibido: 01-06-14; Aprobado: 11-06-14
}

\begin{abstract}
RESUMEN
Las enfermedades infecciosas desatendidas (EID) afectan a más de mil millones de personas en el mundo, y están asociadas con la pobreza, el aislamiento geográfico de las poblaciones, la estigmatización social, la escasez de datos sobre estimaciones de la carga de enfermedad local y mundial (subregistro de la enfermedad), insuficientes recursos políticos y financieros para su control, falta de grupos de presión de parte de la población más vulnerable, escasos fármacos y métodos de diagnóstico. En este artículo se describe la relación entre las EID la pobreza e inequidad, se propone un nuevo concepto de enfermedad en el trópico, la ampliación de la lista de enfermedades en el contexto del Perú que comparten las características de las EID, la escasa disponibilidad de medicamentos y pruebas de diagnóstico para enfrentar adecuadamente estas enfermedades, los aportes que viene haciendo el Instituto Nacional de Salud del Ministerio de Salud del Perú en este contexto, y como consideraciones finales se menciona que la solución para la prevención y control de las EID debe ser integral, con un abordaje desde las determinantes sociales de la salud y en el contexto de la lucha contra la pobreza y la inequidad.
\end{abstract}

Palabras clave: Enfermedades infecciosas; Enfermedades tropicales desatendidas; Políticas públicas de salud (fuente: DeCS BIREME).

\section{ABSTRACT \\ ENFERMEDADES INFECCIOSAS DESATENDIDAS SU RELACIÓN CON LA POBREZA E INEQUIDAD Y LAS ÁREAS TRÓPICALES}

Neglected Infectious Diseases (NID) affect more than one billion people worldwide, and are associated with poverty, geographic isolation of populations, social stigma, lack of precise data on estimates on both the global and local burden of disease (underreporting of the diseases), inadequate financial and political resources to effective control measures, lack of lobbying on behalf of the most vulnerable population, as well as scarce drug and diagnostic methods development. In this article we describe the relationship between NID, poverty and inequality, we propose a new concept of disease in the tropics, expanding the list of diseases that share characteristics with NID in the Peruvian context, discuss the limited availability of drugs and diagnostic tests to properly deal with these diseases, as well as highlight the contributions by the Peruvian National Institute of Health, and as final thoughts, we state that the solution for the prevention and control of NID must include an integrated approach, including the social determinants of health in the context of the fight against poverty and inequality.

Key words: Infectious diseases; Neglected tropical diseases; Health public policy (source: MeSH NLM).
Las enfermedades infecciosas desatendidas (EID) son un grupo de enfermedades infecciosas que afectan a más de mil millones de personas en el mundo, principalmente a las personas más pobres. Interfieren con el desarrollo físico y cognitivo de las personas, conllevando a la enfermedad y la muerte, además de limitar sus oportunidades para la vida y la productividad en el trabajo, constituyéndose en un círculo pernicioso de pobreza y enfermedad. Estas enfermedades han sido descuidadas durante décadas, primero como parte de un desconocimiento general de los países en desarrollo y, más recientemente, debido a la intensidad en la atención de otras enfermedades como el $\mathrm{VIH} /$

Citar como: Cabezas-Sánchez C. Enfermedades infecciosas desatendidas: un permanente reto para la salud pública y la equidad en el Perú. Rev Peru Med Exp Salud Publica. 2014;31(2):326-35. 
SIDA, la tuberculosis y la malaria, que han recibido importantes aportes de la cooperación internacional y de los propios países, para lograr un mejor control ${ }^{(1,2)}$. Las enfermedades infecciosas desatendidas no solo han costado la vidas -muertes evitables- de millones de personas en todo el mundo y han puesto en peligro la salud de millones más de personas, sino que también han frustrado la posibilidad que en muchos pises se alcancen los objetivos del milenio ${ }^{(3)}$.

Son características comunes de las EID la íntima conexión con la pobreza, el aislamiento geográfico de las poblaciones afectadas, la estigmatización social, la escasez de datos sobre estimaciones de la carga de enfermedad local y mundial (subregistro de la enfermedad), insuficientes recursos políticos y financieros para su control, falta de grupos de presión de parte de la población más vulnerable, así como mecanismos de financiación fragmentados y la escasez de especialistas, métodos de diagnóstico y nuevos tratamientos ${ }^{(4)}$.

Las EID se dan en el contexto de los determinantes sociales de la salud, entendidas estas como las circunstancias en que las personas nacen, crecen, viven, trabajan y envejecen, incluido el sistema de salud. Esas circunstancias son el resultado de la distribución del dinero, el poder y los recursos a nivel mundial, nacional y local, que depende a su vez de las políticas adoptadas. Los determinantes sociales de la salud explican la mayor parte de las inequidades sanitarias, esto es, de las diferencias injustas y evitables observadas en y entre los países en lo que respecta a la situación sanitaria ${ }^{(3)}$. Por otro lado, las inaceptables inequidades en salud dentro y entre países no pueden ser abordadas solamente por el sector, sino requieren de soluciones políticas globales. Las normas, políticas y prácticas que derivan de la interacción internacional deben ser entendidas como determinantes políticos de la salud que causan y mantienen inequidades en salud ${ }^{(5)}$.

Se debe mencionar que a las EID se le da también la denominación de enfermedades tropicales desatendidas (ETD) en razón a que son más frecuentes en los trópicos; sin embargo, más que solo un cambio en la denominación, debemos verlas desde otro enfoque para considerarlas más bien como enfermedades del trópico, dado que en estas áreas coinciden con determinantes de pobreza e inequidad y se dan, además de las enfermedades infecciosas, la desnutrición, las enfermedades crónicas y otras condiciones que se encuentran en una sobreposición del perfil epidemiológico, donde, aun manteniéndose las enfermedades infecciosas, empiezan a incrementarse las enfermedades crónicas. Por otro lado, en razón a la predominancia de las EID en poblaciones pobres y en espacios geográficos definidos, debemos considerarlas más bien poblaciones desatendidas, y no solo circunscribirnos a las infecciones y sus etiologías de manera aislada.

Existe cada vez más evidencias que estas enfermedades infecciosas, ahora llamadas desatendidas, han tenido su origen desde los inicios del poblamiento de la tierra por el ser humano. Si bien se conocía que muchas enfermedades infecciosas aparecieron en el periodo neolítico, cuando los animales fueron domesticados por primera vez, o después, existen hallazgos utilizando pruebas de biología molecular que estiman la aparición de estas infecciones desde el paleolítico (hace 2,5 millones de años a 10000 años). Algunas de ellas, como es el caso de las infecciones por Treponema pallidum, Bordetella pertusis, Mycobacterium leprae, Micobacterium tuberculosis, Helycobacter pylori, Taenia solium y Taenia saginata, estarían entre los 5 millones y 2 millones de años, mientras que Micobacteriun bovis estaría en los 10000 años, y la infección por HIV, HTLV, hepatitis $B$, sarampión son los más recientes en su aparición. En estos periodos nuestros ancestros habitaban aún en el África ${ }^{(6)}$. Estos hallazgos nos muestran cómo en el mundo aún hay grupos poblacionales en los que se mantienen estas enfermedades, como son los pobres, los marginados, los pueblos indígenas y minorías étnicas, considerándose a algunas aún como legado de las épocas de esclavitud, por lo que su eliminación es un imperativo moral.

\section{ENFERMEDADES INFECCIOSAS DESATENDIDAS EN EL MUNDO Y SU CONTEXTUALIZACIÓN EN EL PERÚ}

Entre las EID priorizadas por la OMS para la región de las Américas se encuentran (7): la enfermedad de Chagas, el dengue, la echinococcosis (hidatidosis), la fascioliasis, la anquilostomiasis, la leishmaniosis, la lepra, la filariasis linfática, la onchocercosis, otras infecciones transmitidas por el suelo, la schistosomiasis, taeniasis solium y las cisticercosis, el tracoma y la rabia. Considerando las definiciones y características de las enfermedades infecciosas desatendidas (4), es muy probable que en cada país o región se den enfermedades infecciosas que afectan a grupos poblacionales en determinados nichos ecológico-sociales y en áreas geográficas donde las determinantes sociales permiten que estas enfermedades estén presentes tanto en zonas rurales como periurbanas. En ese sentido, en el Perú, además de las enfermedades mencionadas por la OMS y considerando los criterios de enfermedades desatendidas, se puede incluir otras enfermedades 
Tabla 1. Enfermedades infecciosas desatendidas (EID) según clasificación y priorización de la OMS y propuesta de otras enfermedades infecciosas a ser consideradas en el Perú

\begin{tabular}{|c|c|}
\hline EID según clasificación de la OMS ${ }^{(7)}$ & EID que deben ser consideradas en el Perú \\
\hline Virus & Virus \\
\hline Dengue/formas graves de dengue* & Oropouche ${ }^{(19-21)}$ \\
\hline Rabia* & Mayaro ${ }^{(16,17)}$ \\
\hline Bacterias & Encefalitis equina venezolana ${ }^{(20)}$ \\
\hline Úlcera de Buruli & Hantavirus ${ }^{(24,25)}$ \\
\hline Lepra (enfermedad de Hansen)* & Fiebre amarilla ${ }^{(26,27)}$ \\
\hline Tracoma & HTLV-I (28,29) \\
\hline Pian & Hepatitis Ddelta (asociada a HBV) ${ }^{(11-30)}$ \\
\hline Protozoos & Bacterias \\
\hline Enfermedad de Chagas* & Enfermedad de Carrión $(25,26,44)$ \\
\hline Tripanosomiasis africana humana (enfermedad del sueño) & Peste $(27,28,29)$ \\
\hline Leishmaniosis* & Leptospirosis ${ }^{(31,32)}$ \\
\hline Helmintos & Brucelosis $(38,39)$ \\
\hline Cisticercosis/teniasis* & Hongos \\
\hline Dracunculiasis (enfermedad del gusano de Guinea) & Esporotricosis $(40,41)$ \\
\hline Equinococosis* & Paracoccidiodomicosis ${ }^{(42)}$ \\
\hline Trematodiasis transmitidas por los alimentos & Otros \\
\hline Filariasis linfática* & Ofidismo y loxocelismo ${ }^{(43,45)}$ \\
\hline \multicolumn{2}{|l|}{ Oncocercosis (ceguera de los ríos)* } \\
\hline \multicolumn{2}{|l|}{ Esquistosomiasis* } \\
\hline Helmintiasis transmitidas por el suelo* & \\
\hline
\end{tabular}

infecciosas como la enfermedad de Carrión, la peste, la leptospirosis, la brucelosis, el HTLV I-II, la micosis subcutánea como la esporotricosis, las micosis profundas como la paracoccidiodomicosis, la fiebre amarilla, las fiebres hemorrágicas como hantavirus; así mismo, enfermedades virales febriles como el mayaro, el oropouche y la encefalitis equina venezolana, el ofidismo, el loxocelismo y las nuevas presentaciones como la lonomiasis, que cada vez se ve con más frecuencia (Tabla 1). Lo que se quiere expresar es que en razón a las definiciones de EID, se debe dar flexibilidad en cada realidad, como es el caso de Perú, para incluir y abordar el control de estas enfermedades que pueden tener características sui generis en su presentación y ubicación, en el marco de las determinantes que las condicionan.

Estas enfermedades, como mencionamos, se dan en población pobre y en determinadas áreas geográficas, que son nichos ecológico-sociales, donde se dan las condiciones para la presencia no solo de una de ellas, sino además de varias que comparten estos ámbitos de la enfermedad y sus condicionantes. Cuando se ve la presencia y distribución de estas enfermedades se puede correlacionarlas con las áreas de pobreza en el Perú (Figura 1) y los determinantes sociales. La distribución geográfica de las principales enfermedades desatendidas en nuestro país se muestran en las Figuras 2 y 3 .
Así, en la encuesta del INEI 2012, se puede notar que la carencia de agua potable y de desagüe en población pobre es de 35 y $65,9 \%$, mientras que en pobres extremos es de 48 y $89,6 \%$ respectivamente ${ }^{(8)}$. Igualmente, si vemos las características de la vivienda, en los pobres, el material predominante de las paredes en $47 \%$ es adobe/quincha; $15 \%$ de tapia, y $20 \%$ de ladrillo, en comparación al $60,9 \%$ en los no pobres ${ }^{(9)}$; en cuanto al piso, las viviendas de los pobres es de tierra en el $66,8 \%$. En estas viviendas se dan las condiciones para el desarrollo de infecciones como las transmitidas

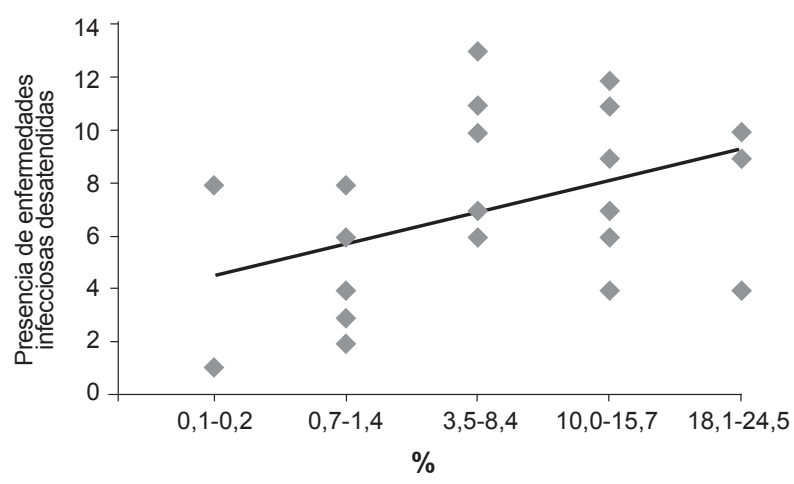

Niveles de pobreza extrema

Figura 1. Correlación entre presencia de enfermedades infecciosas desatendidas y niveles de pobreza extrema en Perú 


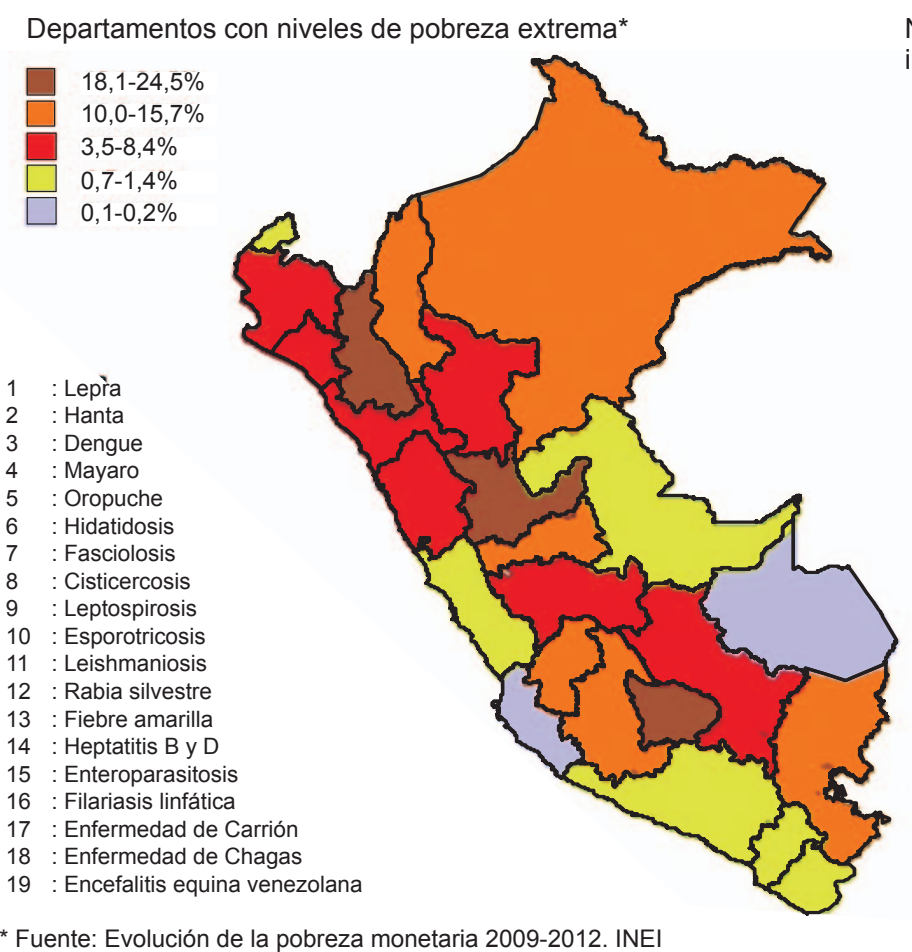

Niveles de pobreza extrema y presencia de enfermedades infecciosas desatendidas según departamentos del Perú

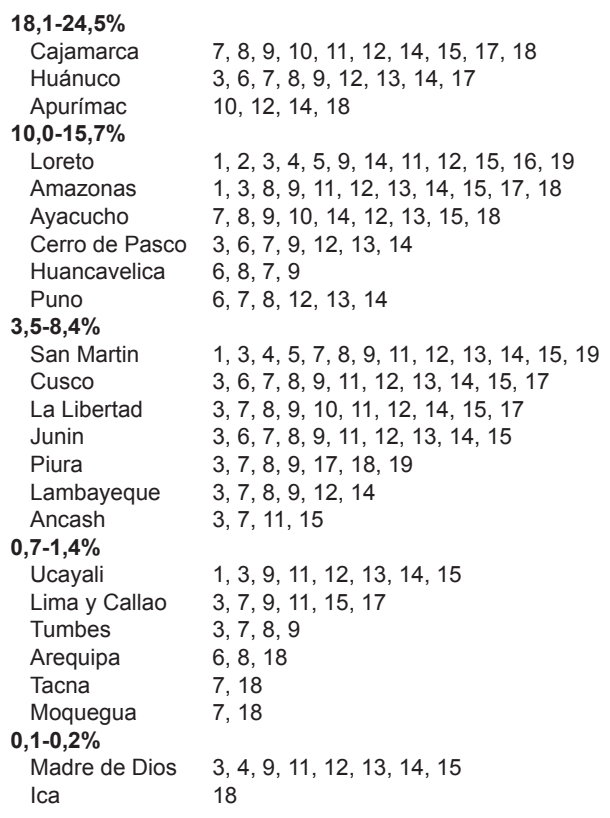

Figura 2. Niveles de pobreza y presencia de enfermedades infecciosas desatendidas según departamentos del Perú

por vectores (enfermedad de Chagas, enfermedad de Carrión, leishmaniosis) y las enteroparasitosis, que se suman a las condiciones del consumo de agua y la eliminación inadecuada de excretas.

Al analizar la incidencia de la pobreza, de acuerdo con la lengua materna de las personas, también se puede notar que está más relacionada con aquella población que tiene como lengua aprendida en su niñez una lengua nativa, como el quechua, el aimara o una lengua amazónica. Así, en el 2013, la pobreza afectó al $35,9 \%$ de las personas que mencionaron tener como lengua materna una lengua nativa, siendo casi el doble que en la población que tiene como lengua materna el castellano $(20,8 \%)$.

Algunos ejemplos de lo mencionado se pueden graficar en poblaciones nativas de laAmazonía peruana, a las que sobre su condición de pobreza y aislamiento geográfico se agregó el problema de la violencia política y militar en décadas pasadas, que los obligó a constituirse en agrupaciones con las más precarias condiciones de vida, insalubridad y carencia de alimentos, donde evidentemente se acentuaron enfermedades como la hepatitis B y delta, enteroparasitosis, malaria, anemia, desnutrición crónica además del trauma y secuelas dejadas por la violencia (Figura 4A). Hay intervenciones en estas comunidades que están tratando de controlar algunos de los problemas como la hepatitis viral $\mathrm{B}^{(11)}$. Sin embargo, también debemos hacer notar que, como resultado de la influencia de las costumbres occidentales, están generándose nuevos problemas como la infección por VIH ${ }^{(12)}$ o la aparición de enfermedades crónicas, cuando aún no se han superado las enfermedades infecciosas ${ }^{(13)}$.

Otro ejemplo de la presencia de las determinantes sociales es el caso de la peste en un valle costero como Chicama, donde existen plantaciones de caña de azúcar, lo cual atrae trabajadores migrantes de la sierra de Cajamarca, y que en su lugar de trabajo viven en condiciones precarias y expuestas a la peste debido a su estrecho contacto con las ratas (Rattus rattus), las pulgas (Xenopsylla cheopis), y la Yersinia pestis $^{(14)}$ (Figura 4B). Estos dos ejemplos nos muestran claramente que las determinantes sociales se dan en territorios definidos, donde residen e interactúan las poblaciones descritas.

Igualmente, se puede apreciar que, ligada a la pobreza monetaria y la inequidad, está el nivel de educación. En la población de pobres, el 48,7\% tiene 


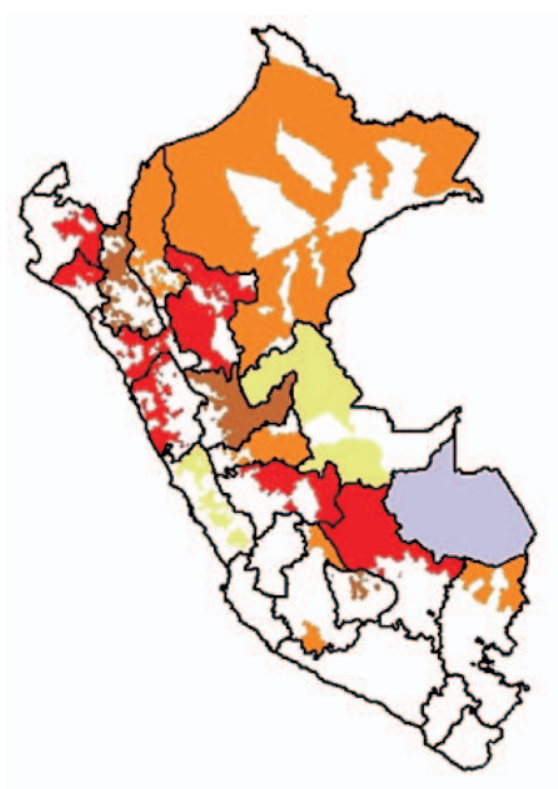

Leishmaniosis 2013

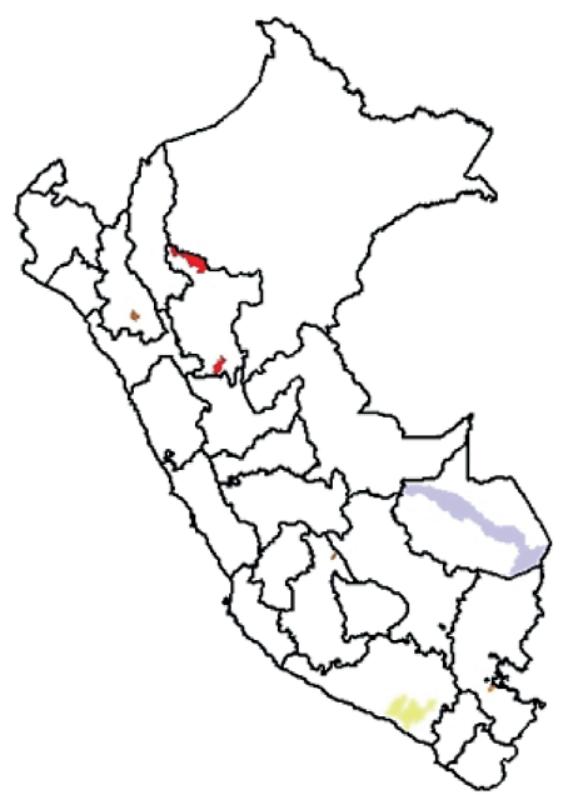

Enfermedad de Chagas 2013

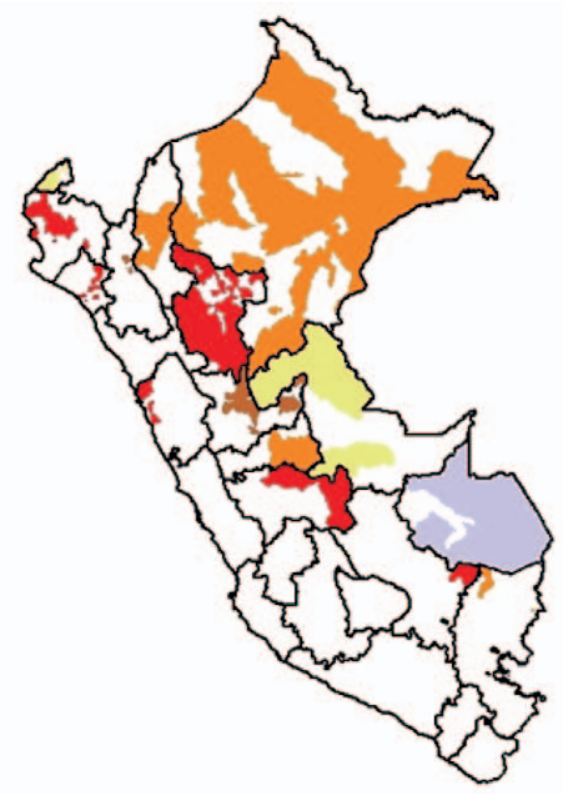

Dengue 2013

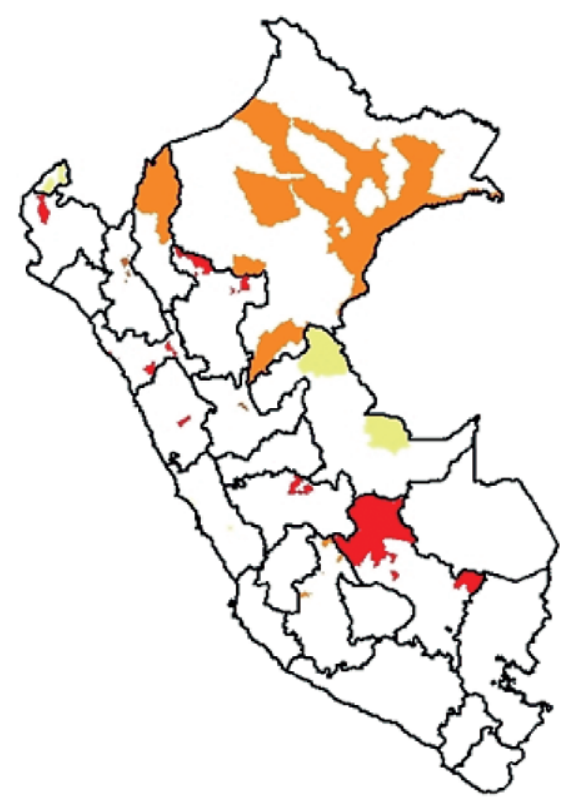

Leptospirosis 2013

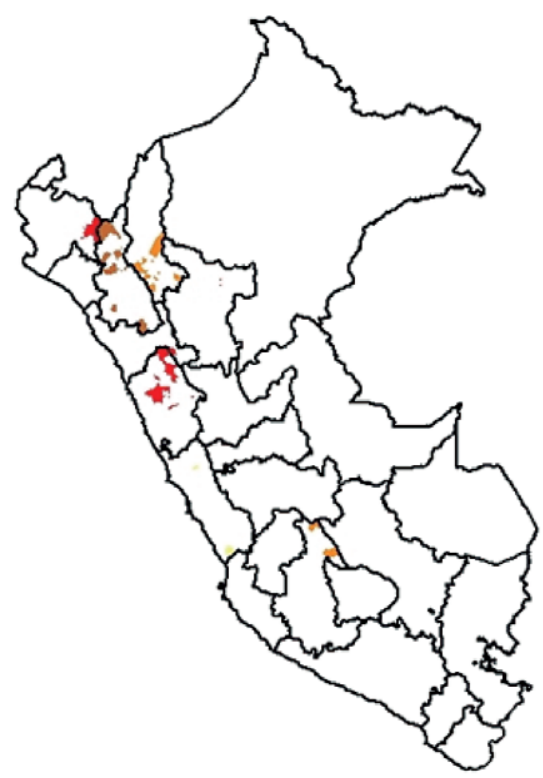

Enfermedad de Carrión 2013

Niveles de pobreza extrema

$18,1-24,5 \%$

$10,0-17,7 \%$

$3,5-8,4 \%$

$0,7-1,4 \%$

$0,1-0,2 \%$

Figura 3. Presencia de casos de leishmaniosis, dengue, enfermedad de Carrión, enfermedad de Chagas y leptospirosis según los niveles de pobreza de las regiones en el Perú 


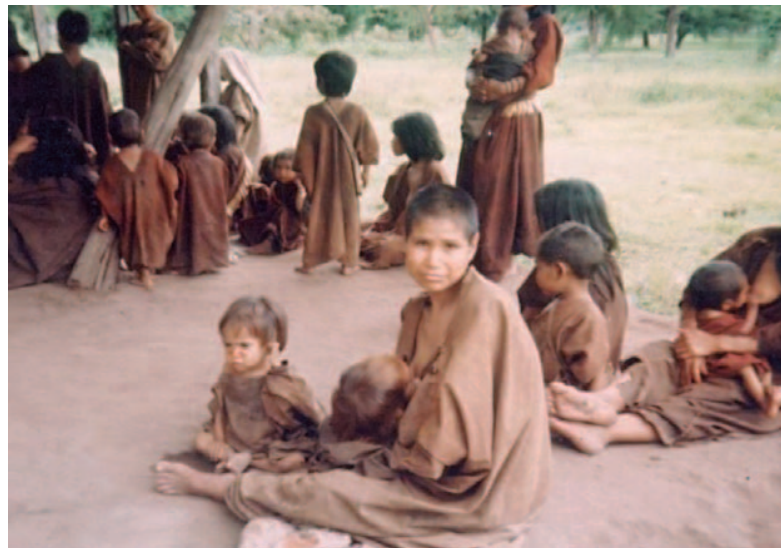

Nativos asháninkas, concentrados en grupos de extrema pobreza, en la selva de Junín

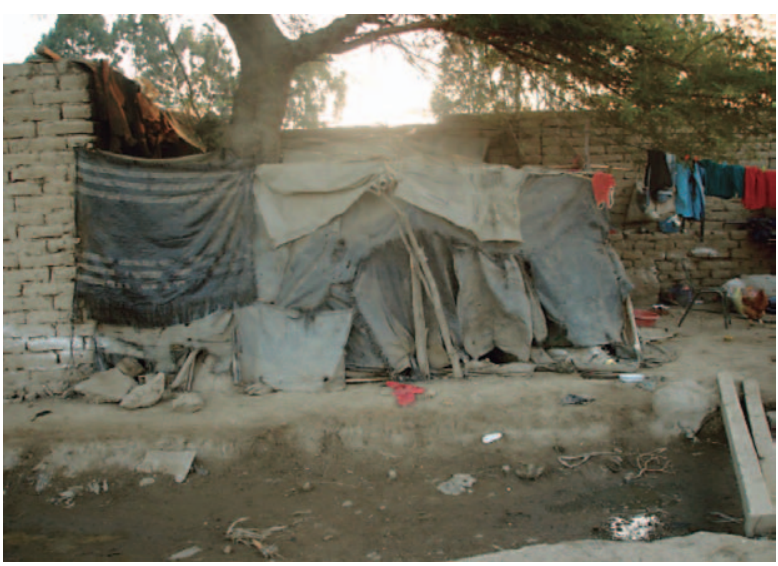

Vivienda precaria de migrantes pobres de Cajamarca al valle de Chicama (La Libertad)

Figura 4. Determinantes sociales de la salud en diferentes contextos

educación primaria y el $43,5 \%$ educación secundaria. En la población de pobres extremos, el $66,9 \%$ tiene educación primaria y el 30,3\% educación secundaria; mientras que en los no pobres, el 19,9\% tiene educación primaria y $43,6 \%$ educación secundaria. Esta situación también contribuye a la presencia de enfermedades infecciosas desatendidas en las zonas donde residen estas poblaciones ${ }^{(8)}$.

\section{LOS MEDICAMENTOS Y PRUEBAS DE DIAGNÓSTICOPARAENFERMEDADES INFECCIOSAS DESATENDIDAS}

Para enfrentar las enfermedades infecciosas desatendidas se requieren, entre otros, la disponibilidad de medicamentos que sean eficaces, asequibles y fáciles de usar; sin embargo, estos son insuficientes $y$, en otros casos, casi ausentes. Pese a que la ciencia y la tecnología han avanzado lo suficiente como para proporcionar los medicamentos necesarios, son muy pocos los nuevos fármacos desarrollados. Esto se debe a que la rentabilidad económica para la investigación y desarrollo de nuevos medicamentos para EID no es muy atractiva, como sí lo son para enfermedades crónicas cada vez más prevalentes. Así, el estudio realizado por Trouiller et al. (15) muestra que de las 1393 nuevas presentaciones químicas comercializadas entre 1975 y 1999, solo 16 eran para enfermedades tropicales y la tuberculosis. Hay 13 veces mayor probabilidad que un fármaco sea lanzando al mercado para los trastornos del sistema nervioso central o el cáncer, que para una enfermedad desatendida. La industria farmacéutica argumenta que la investigación y el desarrollo son demasiado costosos y es arriesgado invertir en enfermedades desatendidas de bajo rendimiento. Existen iniciativas que han tratado de superar esta limitación del mercado a través de paquetes de estímulos y la colaboración público-privada.

La única forma de revertir esta situación es la decisión política de los países y el desarrollo de estrategias nuevas y creativas para estimular el desarrollo de medicamentos para las EID con participación pública y estimulando al sector privado, priorizando la salud como un derecho sobre los intereses puramente económicos. Para este efecto, creemos importante concretar los acuerdos y convenios entre los países que comparten el problema de las EID, para que a través de la cooperación se pueda contar con la producción de medicamentos esenciales. En el caso del Perú, como un paso inicial y a través de la cooperación entre el INS y la Fundación Oswaldo Cruz del Brasil, se está implementando un proceso de transferencia tecnológica inversa para la producción de medicamentos antimaláricos.

Algo similar a los medicamentos ocurre con las pruebas de diagnóstico, donde igualmente es muy limitada la investigación, el desarrollo y la innovación, por lo tanto su disponibilidad. Hay esfuerzos que vienen haciendo algunas organizaciones como el TDR-OMS ${ }^{(16)}$ y los países como Brasil ${ }^{(17,18)} y$, de algún modo, Perú, como veremos más adelante. Entre los países que compartimos las EID, hay aún un camino común por donde transitar, incluyendo el desarrollo de materia prima para la producción de medicamentos cuya patente ya venció, así como la investigación y el desarrollo de nuevas pruebas de diagnóstico y medicamentos, considerando que para los grandes productores los medicamentos para las EID ya no 


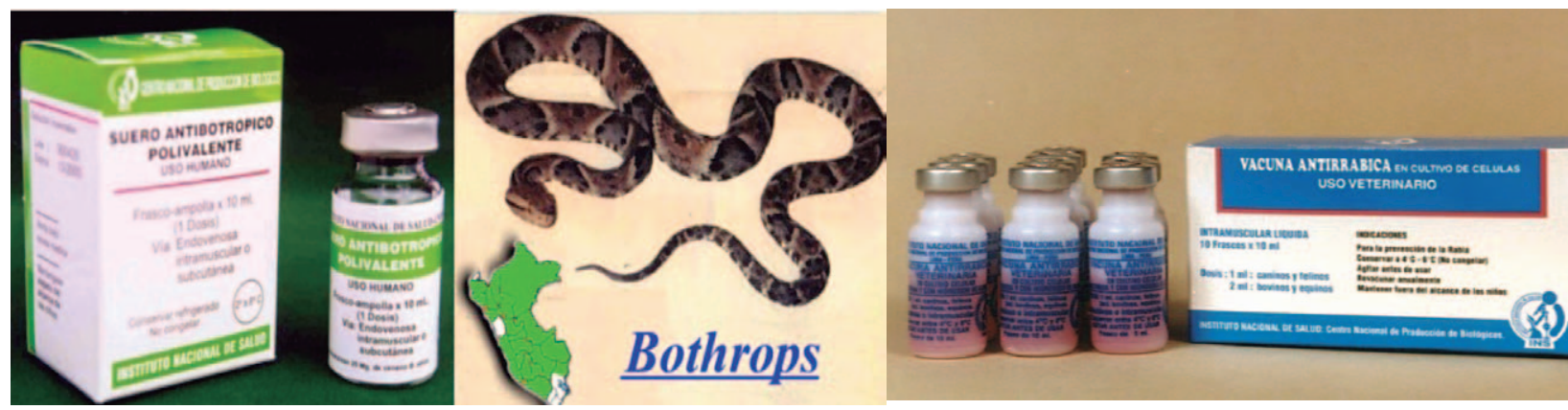

Figura 5. Suero antibotrópico polivalente y vacuna antirrábica, producidos por el Centro Nacional de Productos Biológicos del Instituto Nacional de Salud del Perú

son atractivas. Es un reto para los países y la Red de Institutos Nacionales de Salud (RINS, IANPHI) el catalizar estos procesos.

\section{APORTES DEL INSTITUTO NACIONAL DE SALUD DEL PERÚ Y PERSPECTIVAS}

En 1896, cuando el Instituto Vaccinal de Lima inició la producción de la vacuna antivariólica para la erradicación de la viruela en el Perú, enfermedad que diezmaba a los peruanos de todas las clases y razas, pero particularmente a la indígena, se marcó el primer hito de lo que sería el Instituto Nacional de Salud (INS) y su papel de contribución a la solución de problemas sanitarios, desde los albores de la época republicana. Posteriormente, y hasta la actualidad, se producen biológicos como el suero antibotrópico polivalente, el suero antiloxocélico, las vacunas de virus inactivados contra la rabia (Figura 5), y pruebas para el diagnóstico de brucelosis. Así mismo, cuenta con las capacidades para el diagnóstico referencial de todas las EID que figuran en la Tabla 1.

En los últimos tres años, producto de investigaciones previas en el Centro Nacional de Salud Pública del INS, se ha dado inicio a la producción de pruebas de diagnóstico a través del Centro de Producción de
Biológicos del mismo INS, en una serie llamada Tariki® (del kechua: te encontré). Los primeros productos son un kit de ELISA para determinar anticuerpos IgM para el diagnóstico de dengue y de manera similar para la fiebre amarilla (Figura 6). Si bien existen en el mercado mundial kits de ELISA para el diagnóstico serológico de dengue, no hay disponibilidad en el mercado de kits para el diagnóstico de fiebre amarilla, y el producido por el INS es el primero desarrollado como kit termoestable, ¿cómo explicar esto último?. Evidentemente no es por la falta de tecnologías ni capacidades en los países industrializados para su producción, sino porque su producción no tiene rentabilidad económica. Sin embargo, debido a su alta rentabilidad social lo asume el Estado a través del INS, dado que la fiebre amarilla, así como otras EID, sigue constituyendo un problema de salud pública, particularmente en población pobre de la sierra que migra a la selva alta en busca de mejores condiciones de vida.

Antes de contar con estos kits, se tenían que enviar a los laboratorios regionales de salud pública, insumos para pruebas in house con muchas dificultades logísticas y riesgos de alterar la calidad de las pruebas y sus resultados. Actualmente, se cuentan con estos kits Tariki $^{\circledast}$ que tienen alta sensibilidad y especificidad; pueden ser mantenidas sin problemas a $4{ }^{\circ} \mathrm{C}$, y son
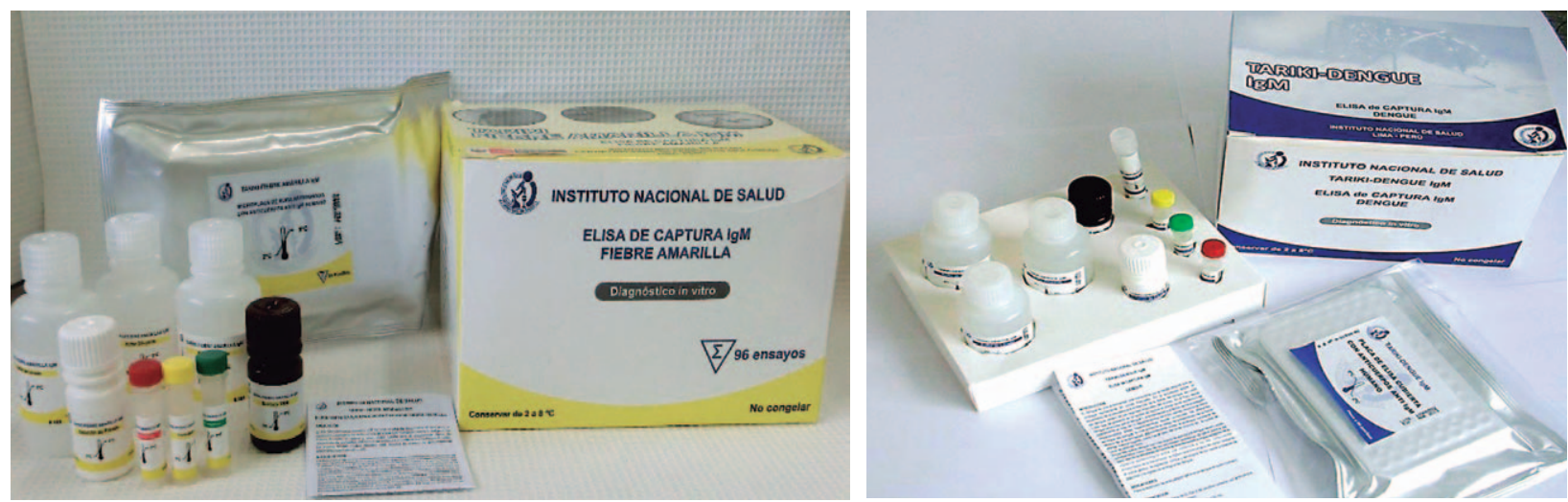

Figura 6. Kits de ELISA (Tariki®) para el diagnóstico de dengue y fiebre amarilla 
distribuidos a los laboratorios de las regiones de manera oportuna, se debe destacar el menor costo de estas pruebas respecto a las disponibles en el mercado.

En el caso del Perú, este tipo de kits ayudan a solucionar el problema del diagnóstico de manera descentralizada, allí en el nicho ecológico-social (hábitat donde está el reservorio del virus de la fiebre amarilla y el vector, solo falta el ingreso de una persona susceptible) donde ocurre la enfermedad, permitiendo un diagnóstico oportuno para el manejo individual de los casos, pero también para orientar las medidas de control oportunas en caso de brotes epidémicos. Consideramos esto una muestra del desarrollo y el uso de tecnologías para contribuir a la prevención, control y, eventualmente, la eliminación de enfermedades desatendidas que se dan en nuestros países. Existe la opción de compartir estos instrumentos con los institutos nacionales de salud de los países latinoamericanos y de otros continentes como el África. Asimismo, están en curso el desarrollo de otras pruebas para el diagnóstico de leptospirosis, leishmaniosis, enfermedad de Chagas, hidatidosis, entre otras.

Con la finalidad de dar respuesta oportuna en el diagnóstico de las EID, el INS ha conformado una Red Nacional de Laboratorios de Salud Pública para acercar el diagnóstico a las zonas donde están los problemas, red que debe ser fortalecida en sus capacidades con la transferencia de tecnologías desarrolladas en el nivel referencial. Como se mencionó líneas arriba, producto de la cooperación bilateral entre el INS y la Fundación Oswaldo Cruz, se viene implementando la transferencia tecnológica inversa, para la producción de medicamentos antimaláricos. Este es el inicio de un proceso de cooperación horizontal para ampliar a la producción de medicamentos y kits de diagnóstico para EID que son comunes en los países de región sudamericana y del África. Para lograrlo, es importante participar de las redes nacionales e internacionales de investigación, desarrollo e innovación de tecnologías sur-sur y norte-sur. Al considerar un abordaje integral de las EID y sus determinantes se puede contribuir a reducir las brechas de inequidad, que tienen su máxima expresión en poblaciones afectadas por las EID.

El control y la eliminación de las enfermedades infecciosas desatendidas pueden ser indicadores muy sensibles para medir el impacto de la reducción de la pobreza y de la cobertura universal de salud. Son representativos de cómo los países en desarrollo se preocupan por la salud de los sectores más pobres de la población ${ }^{(46)}$. Es de esperar que la reducción de la pobreza en nuestro país pueda tener impacto en el control de enfermedades infecciosas desatendidas, aunque esta reducción es más lenta en áreas rurales
(5,2\% del 2009 al 2012), en comparación a áreas urbanas (18,7\% del 2009) según el INEI (47).

\section{CONSIDERACIONES FINALES}

La solución que se plantea para la prevención y control de las EID es integral, con un abordaje desde las determinantes sociales de la salud, que incluyan estrategias interprogramáticas y acciones intersectoriales con educación, vivienda, desarrollo, agua y saneamiento básico, medioambiente, agricultura y ganadería, necesarias para modificar los determinantes sociales y ambientales de estas enfermedades ${ }^{(48,49)}$.

En ese contexto se debe considerar las recomendaciones de la OMS que incluye cinco estrategias para la prevención, control, eliminación y erradicación de las enfermedades tropicales desatendidas: quimioterapia preventiva, que tiene por objeto la optimización del uso a gran escala de medicamentos en una sola dosis, actualmente contra cuatro helmintiasis (filariasis linfática, oncocercosis, schistosomiasis, y helmintiasis transmitidas por el suelo); la intensificación del tratamiento y manejo de casos, como la tripanosomiasis africana, la leishmaniosis, la enfermedad de Chagas y la úlcera de Buruli, procurando atención especializada y descentralizada a través de la mejora de detección de casos, con el objetivo de prevenir la mortalidad, reducir la morbilidad e interrumpir la transmisión; el control de vectores y reservorios intermediarios, el cual debe ser integrado; incluir la salud pública veterinaria, considerando que varias de las EID son zoonosis; finalmente, el suministro de agua, el saneamiento adecuado y la higiene ${ }^{(50)}$.

La persistencia del "círculo vicioso" entre la pobreza y la mala salud demuestra la importancia de vincular las actividades del sector salud con las de otros sectores como educación, vivienda, agua y saneamiento, trabajo, agricultura, medioambiente e industria, considerando los derechos humanos y su normatividad, todo enmarcado en claros objetivos de desarrollo. Consideramos que el modelo intersectorial, como se viene actualmente afrontando en nuestro país la desnutrición crónica y la anemia, son estrategias que muy bien deben hacerse extensivos a las enfermedades infecciosas desatendidas en el marco de la lucha contra la pobreza y la inequidad, a la cual están estrechamente asociadas.

Hay muchos esfuerzos e iniciativas en los países y a nivel internacional para enfrentar las EID, como los de la OMS y la OPS, los acuerdos entre diferentes actores, incluyendo representantes de la industria farmacéutica, de organizaciones financieras, de investigación y de 
desarrollo (51). Todo ello es necesario; sin embargo, la lucha contra las EID también debe estar anclada en los objetivos de desarrollo sostenible pos-2015, junto con la lucha contra la pobreza y por la reducción sostenida de las inequidades. Finalmente, es importante considerar que la gobernanza global para la salud debe basarse en compromisos de solidaridad mundial y de responsabilidad compartida; el desarrollo sostenible y saludable para todos requiere de un sistema económico y político global que sirva a una comunidad mundial de personas saludables en un mundo saludable.

Agradecimientos: a los doctores Víctor Suárez y Hugo Arroyo, por sus valiosos aportes y comentarios en la elaboración del presente artículo.

\section{REFERENCIAS BIBLIOGRÁFICAS}

1. Centers for Disease Control and Prevention. Neglected Tropical Diseases [Internet]. Atlanta: CDC; [citado el 17 de abril del 2014]. Disponible en: http://www.cdc.gov/ globalhealth/ntd/

2. Feasey N, Wansbrough-Jones M, Mabey D, Solomon A. Neglected tropical diseases. Br Med Bull. 2010;93(1):179200.

3. World Health Organization. First WHO report on neglected tropical diseases working to overcome the global impact of neglected tropical diseases. Geneva: WHO; 2010.

4. Utzinger J, Becker SL, Knopp S, Blum J, Neumayr AL, Keiser J, et al. Neglected tropical diseases: diagnosis, clinical management, treatment and control. Swiss Med Wkly. 2012 Nov 22;142:w13727. doi: 10.4414/ smw.2012.13727.

5. Ottersen OP, Dasgupta J, Blouin C, Buss $\mathrm{P}$, Chongsuvivatwong V, Frenk J, et al. The political origins of health inequity: prospects for change. Lancet. $2014 \mathrm{Feb}$ 15;383(9917):630-67. doi: 10.1016/ S0140-6736(13)62407-1.

6. Trueba G, Dunthorn M. Many neglected tropical diseases may have originated in the paleolithic or before: new insights from genetics. PLoS Negl Trop Dis. 2012;6(3):e1393. doi: 10.1371/ journal.pntd.0001393.

7. World Health Organization. The 17 neglected tropical diseases [Internet]. Geneva: WHO; 2014 [citado el 17 de abril del 2014]. Disponible en: http:// www.who.int/neglected_diseases/ diseases/en/

8. Instituto Nacional de Estadística e Informática. Perú: Encuesta Nacional de Hogares sobre Condiciones de Vida y Pobreza 2012 [Internet]. Lima: INEI; 2012 [citado el 17 de abril del 2014]. Disponible en: http://webinei.inei.gob. pe/anda_inei/index.php/catalog/195
9. Instituto Nacional de Estadística e Informática. Citas de pobreza 2013 [Internet]. Lima: INEI; [citado el $17 \mathrm{de}$ abril del 2014]. Disponible en: http:// www.inei.gob.pe/cifras-de-pobreza/

10. Cabezas C, Suárez M, Romero G, Carrillo C, García MP, Reátegui J, et al. Hiperendemicidad de hepatitis viral B y Delta en pueblos indígenas de la Amazonía Peruana. Rev Peru Med Exp Salud Publica. 2006 Abr;23(2):114-22.

11. Cabezas-Sánchez C, Trujillo Villarroel O, Zavaleta Cortijo C, Culqui-Lévano D, Suarez-Jara M, Cueva-Maza N, Monzon S. Prevalencia de la infección por el virus de hepatitis b en niños menores de 5 años de comunidades indígenas de la amazonía peruana posterior a intervenciones mediante inmunización. Rev Peru Med Exp Salud Publica. 2014; 31(2):

12. Bartlett EC, Zavaleta $\mathrm{C}$, Fernández $\mathrm{C}$, Razuri H, Vilcarromero S, Vermund $\mathrm{SH}$, et al. Expansion of HIV and syphilis into the Peruvian Amazon: a survey of four communities of an indigenous Amazonian ethnic group. Int J Infect Dis. 2008 Nov;12(6):e89-94. doi: 10.1016/j.jiid.2008.03.036.

13. Romero C, Zavaleta C, Cabrera L, Gilman RH, Miranda JJ. Hipertensión arterial y obesidad en indígenas asháninkas de la región Junín, Perú. Rev Peru Med Exp Salud Publica. 2014;31(1):78-83.

14. Pachas P, Mendoza L, González D, Fernández V, Céspedes M. Control de la peste en La Libertad, Perú. Rev Peru Med Exp Salud Publica. 2010;27(3):473-7.

15. Trouiller P, Olliaro P, Torreele E, Orbinski J, Laing R, Ford N. Drug development for neglected diseases:a deficient market and a public-health policy failure. Lancet. 2002 Jun 22; 359(9324):2188-94.

16. TDR/WHO For research on diseases of poverty. Diagnostics [Internet]. Geneva: WHO; [citado el 17 de abril del 2014]. Disponible en: http://www.who.int/ tdr/diseases-topics/diagnostics/en/
17. Utzinger J, Becker SL, Knopp S, Blum J, Neumayr AL, Keiser J, et al. Neglected tropical diseases: diagnosis, clinical management, treatment and control. Swiss Med Wkly. 2012 Nov 22;142:w13727. doi: 10.4414/smw.2012.13727.

18. Morel CM, Serruya SJ, Penna GO, Guimarães R. Co-authorship network analysis: a powerful tool for strategic planning of research, development and capacity building programs on neglected diseases. PLoS Negl Trop Dis. 2009 Aug 18;3(8):e501. doi: 10.1371/journal. pntd.0000501.

19. Baisley KJ, Watts DM, Munstermann LE, Wilson ML. Epidemiology of endemic Oropouche virus transmission in upper Amazonian Peru. Am J Trop Med Hyg. 1998 Nov;59(5):710-6.

20. Watts DM, Lavera V, Callahan J, Rossi C, Oberste MS, RoehrigJT, et al. Venezuelan equine encephalitis and Oropouche virus infections among Peruvian army troops in the Amazon region of Peru. Am J Trop Med Hyg. 1997 Jun;56(6):661-7.

21. Watts DM, Phillips I, Callahan JD, Griebenow W, Hyams KC, Hayes CG. Oropouche virus transmission in the Amazon River basin of Peru. Am J Trop Med Hyg. 1997 Feb;56(2):148-52.

22. Halsey ES, Siles C, Guevara C, Vilcarromero S, Jhonston EJ, Ramal C, et al. Mayaro virus infection, Amazon Basin region, Peru, 2010-2013. Emerg Infect Dis. 2013 Nov;19(11):1839-42. doi: 10.3201/eid1911.130777.

23. Tesh RB, Watts DM, Russell KL, Damodaran C, Calampa C, Cabezas C, et al. Mayaro virus disease: an emerging mosquito-borne zoonosis in tropical South America. Clin Infect Dis. 1999 Jan; 28(1):67-73.

24. Jhonston EJ, Casanova W, RodriguezFerrucci H. Hantavirosis: algunas consideraciones de esta nueva infección en el Perú. Rev Peru Med Exp Salud Publica. 2012 Jul-Sep; 29(3):414-5. 
25. Casapía M, Mamani E, García MP, Miraval ML, Valencia P, Quino AH, et al. Síndrome pulmonar por Hantavirus (virus río Mamoré) en la Amazonía Peruana. Rev Peru Med Exp Salud Publica. 2012 Jul-Sep; 29(3):390-5.

26. Bryant J, Wang H, Cabezas C, Ramirez G, Watts D, Russell K, et al. Enzootic transmission of yellow fever virus in Peru. Emerg Infect Dis. 2003 Aug; 9(8):92633.

27. Perú, Ministerio de Salud, Dirección General de Epidemiologia. Fiebre amarilla selvática: Perú 2013 [Internet]. Lima: MINSA; 2013 [citado el 17 de abril del 2014]. Disponible en: http:// www.dge.gob.pe/vigilancia/sala/2013/ SE44/f_amarilla.pdf

28. Ita F, Mayer EF, Verdonck K, Gonzalez E, Clark D, Gotuzzo E. Human T-lymphotropic virus type 1 infection is frequent in rural communities of the southern Andes of Peru. Int J Infect Dis. 2014 Feb; 19:46-52. doi: 10.1016/j. ijid.2013.10.005.

29. Ticona E, Huaman MA, Yanque O, Zunt JR. HIV and HTLV-1 coinfection: the need to initiate antiretroviral therapy. J Int Assoc Provid AIDS Care. 2013 Nov-Dec;12(6):373-4. doi: $10.1177 / 2325957413500988$.

30. Cabezas C, Gotuzzo E, Escamilla J, Philips I. [Prevalence of serological markers of viral hepatitis $A, B$ and delta in

apparently healthy schoolchildren of Huanta, Peru]. Rev Gastroenterol Peru. 1994 May-Aug;14(2):123-34. [Article in Spanish].

31. Perú, Ministerio de Salud, Dirección General de Epidemiologia. Enfermedad de Carrión: Perú 2014 [Internet]. Lima: MINSA; 2013 [citado el 17 de abril del 2014]. Disponible en: http://www. dge.gob.pe/portal/docs/vigilancia/ sala/2014/SE22/carrion.pdf

32. Pachas P. Enfermedad de Carrión (bartonelosis) en el Perú. Lima: MINSA; 2001.

33. Cáceres O, Montenegro J, Padilla C, Tarazona D, Bailón H, García P, et al. Whole-Genome Sequencing and Comparative Analysis of Yersinia pestis, the Causative Agent of a Plague Outbreak in Northern Peru. Genome Announc. 2013 Jan;1(1). pii: e00249-12. doi: 10.1128/genomeA.00249-12. Epub 2013 Feb 28.

34. Pachas PE, Mendoza L, González D, Fernández V, Céspedes M. Control de la peste en La Libertad, Perú. Rev Peru Med Exp Salud Publica. 2010 JulSep;27(3):473-7.

35. Donaires LF, Céspedes M, Valencia P, Salas JC, Luna ME, Castañeda A, et al. Peste neumónica primaria con transmisión intrahospitalaria en La Libertad, Perú 2010. Rev Peru Med Exp Salud Publica. 2010 Jul-Sep; 27(3):32636.

36. Donaires LF, Céspedes MJ, Sihuincha MG, Pachas PE. Determinantes ambientales y sociales para la reemergencia de la leptospirosis en la región amazónica del Perú, 2012. Rev Peru Med Exp Salud Publica. 2012 AbrJun; 29(2):280-4.

37. Johnson MA, Smith H, Joeph P, Gilman RH, Bautista CT, Campos $\mathrm{KJ}$, et al. Environmental exposure and leptospirosis, Peru. Emerg Infect Dis. 2004 Jun; 10(6):1016-22.

38. Román K, Castillo R, Gilman RH, Calderón M, Vivar A, Céspedes M, et al. A foodborne outbreak of brucellosis at a police station cafeteria, Lima, Peru. Am J Trop Med Hyg. 2013 Mar; 88(3):552-8. doi: 10.4269/ajtmh.12-0606.

39. Mendoza-Núñez M, Mulder M, Franco MP, Maas KS, Castañeda ML, Bonifacio $\mathrm{N}$, et al. Brucellosis in household members of Brucella patients residing in a large urban setting in Peru. Am J Trop Med Hyg. 2008 Apr;78(4):595-8.

40. Pappas PG, Tellez I, Deep AE, Nolasco D, Holgado W, Bustamante B. Sporotrichosis in Peru: description of an area of hyperendemicity. Clin Infect Dis. $2000 \mathrm{Jan} ; 30(1): 65-70$.

41. Cabezas C, Bustamante B, Holgado W, Begue RE. Treatment of cutaneous sporotrichosis with one daily dose of potassium iodide. Pediatr Infect Dis J. 1996 Apr;15(4):352-4.

42. Burstein Z. Aspectos clínicos de la blastomicosis sudamericana (paracoccidioidomicosis) en el Perú. Rev Peru Med Exp Salud Publica. 2002 EneMar;19(1):43-7.

43. Núñez V, Cid P, Sanz L, De La Torre P, Angulo Y, Lomonte B, et al. Snake venomics and antivenomics of Bothrops atrox venoms from Colombia and the Amazon regions of Brazil, Perú and Ecuador suggest the occurrence of geographic variation of venom phenotype by a trend towards paedomorphism. J Proteomics. 2009 Nov 2;73(1):57-78. doi: 10.1016/j.jprot.2009.07.013.
44. Sanchez Clemente N, Ugarte-Gil CA, Solórzano N, Maguiña C, Pachas P, Blazes D, et al. Bartonella bacilliformis: a systematic review of the literature to guide the research agenda for elimination. PLoS Negl Trop Dis. 2012;6(10):e1819. doi: 10.1371/journal.pntd.0001819.

45. Mendoza A, Cabezas C. Loxoscelismo: evaluación clínica, tratamiento y prevención. Revista Peruana de Enfermedades Infecciosas y Tropicales. 2006;5(1):2-8.

46. Holmes P; WHO Strategic and Advisory Group on Neglected Tropical Diseases. Neglected tropical diseases in the post2015 health agenda. Lancet. 2014 May 24;383(9931):1803. doi: 10.1016/ S0140-6736(14)60875-8.

47. Instituto Nacional de Estadística e Informática. Evolución de la pobreza monetaria, 2009-2012: Informe Técnico. Lima: INEI; 2014.

48. Organización Panamericana de la Salud, Organización Mundial de la Salud. Enfoque integrado de las enfermedades infecciosas desatendidas [Internet]. Washington DC: WHO; 2013 [citado el 17 de abril del 2014]. Disponible en: https://www.paho.org/hq./index. php?option $=$ com_docman $\&$ task $=$ cat view\&gid $=1378 \&$ Itemid $=270 \&$ lang $=\mathrm{e}$ $\mathrm{n}$

49. Holveck JC, Ehrenberg JP, Ault SK, Rojas R, Vasquez J, Cerqueira MT, et al. Prevention, control, and elimination of neglected diseases in the Americas: Pathways to integrated, interprogrammatic, inter-sectoral action for health and development. BMC Public Health. 2007, Jan 17;7:6.

50. World Health Organization. Accelerating work to overcome the global impact of neglected tropical diseases - A roadmap for implementation. Geneva: WHO; 2012.

51. Neglected tropical diseases: becoming less neglected. Lancet. 2014 Apr 12;383(9925):1269. doi: 10.1016/ S0140-6736(14)60629-2.

Correspondencia: César Cabezas Sánchez Dirección: Jr. Cápac Yupanqui 1400 Lima, 11 Perú

Teléfono: 511-7480000 Anexo 2112

Correoelectrónico: ccabezas@ins.gob.pe 